Article

\title{
A Novel Four-Stage Method for Vegetation Height Estimation with Repeat-Pass PolInSAR Data via Temporal Decorrelation Adaptive Estimation and Distance Transformation
}

\author{
Cheng Xing ${ }^{1}{ }^{\oplus}$, Tao Zhang ${ }^{1}{ }^{\oplus}$, Hongmiao Wang ${ }^{1}$, Liang Zeng ${ }^{1}$, Junjun Yin ${ }^{2}$ and Jian Yang ${ }^{1, *}$ \\ 1 Department of Electronic Engineering, Rohm Building, Tsinghua University, Beijing 100084, China; \\ xingc18@mails.tsinghua.edu.cn (C.X.); zhangtao8902@mail.tsinghua.edu.cn (T.Z.); \\ wanghm17@mails.tsinghua.edu.cn (H.W.); zengliang14@mails.tsinghua.edu.cn (L.Z.) \\ 2 School of Computer and Communication Engineering, University of Science and Technology Beijing, \\ Beijing 100083, China; junjun_yin@ustb.edu.cn \\ * Correspondence: yangjian_ee@mail.tsinghua.edu.cn
}

check for updates

Citation: Xing, C.; Zhang, T.; Wang, H.; Zeng, L. Yin, J.; Yang, J. A Novel Four-Stage Method for Vegetation Height Estimation with Repeat-Pass PolInSAR Data via Temporal Decorrelation Adaptive Estimation and Distance Transformation. Remote Sens. 2021, 13, 213. https://doi.org/ $10.3390 /$ rs13020213

Received: 26 November 2020

Accepted: 6 January 2021

Published: 9 January 2021

Publisher's Note: MDPI stays neutral with regard to jurisdictional clai$\mathrm{ms}$ in published maps and institutional affiliations.

Copyright: () 2021 by the authors. Licensee MDPI, Basel, Switzerland. This article is an open access article distributed under the terms and conditions of the Creative Commons Attribution (CC BY) license (https:// creativecommons.org/licenses/by/ $4.0 /)$.

\begin{abstract}
Vegetation height estimation plays a pivotal role in forest mapping, which significantly promotes the study of environment and climate. This paper develops a general forest structure model for vegetation height estimation using polarimetric interferometric synthetic aperture radar (PolInSAR) data. In simple terms, the temporal decorrelation factor of the random volume over ground model with volumetric temporal decorrelation (RVoG-vtd) is first modeled by random motions of forest scatterers to solve the problem of ambiguity. Then, a novel four-stage algorithm is proposed to improve accuracy in forest height estimation. In particular, to compensate for the temporal decorrelation mainly caused by changes between multiple observations, one procedure of temporal decorrelation adaptive estimation via Expectation-Maximum (EM) algorithm is added into the novel method. On the other hand, to extract the features of amplitude and phase more effectively, in the proposed method, we also convert Euclidean distance to a generalized distance for the first time. Assessments of different algorithms are given based on the repeat-pass PolInSAR data of Gabon Lope Park acquired in AfriSAR campaign of German Aerospace Center (DLR). The experimental results show that the proposed method presents a significant improvement of vegetation height estimation accuracy with a root mean square error (RMSE) of $6.23 \mathrm{~m}$ and a bias of $1.28 \mathrm{~m}$ against LiDAR heights, compared to the results of the three-stage method (RMSE: $8.69 \mathrm{~m}$, bias: $4.81 \mathrm{~m}$ ) and the previous four-stage method (RMSE: $7.72 \mathrm{~m}$, bias: $-2.87 \mathrm{~m}$ ).
\end{abstract}

Keywords: vegetation height; forest vertical structure; PolInSAR; RVoG+vtd model; four-stage algorithm; EM algorithm

\section{Introduction}

As the importance of forest in earth system attracts increasing attention, the inversion of forest parameters is gradually becoming a research hotspot. In particular, polarimetric interferometric synthetic aperture radar (PolInSAR) images are widely used in forest height inversion because of its global coverage and low weather sensitivity as well as its ability to extract scattering information and height information simultaneously $[1,2]$. In the ideal case, forest height can be reflected by the phase difference between the volumeonly and ground-dominant polarization states [3]. To extract the phase centers, optimal polarimetric coherence algorithm [3], estimating signal parameter via rotational invariance techniques (ESPRIT) algorithm [4], and model-based target scattering decomposition [5,6] have been developed to effectively extract the phase centers. However, to some extent, the forest vertical structure and wave extinction can affect the estimation of phase centers. Based on the homogeneous hypothesis, Treuhaft et al. proposed a two-layer physical model named the random volume over ground (RVoG) model $[7,8]$. The model contained 
some unknown parameters including forest height, wave extinction, ground phase, and ground-volume ratio, which means a nonlinear optimization should be conducted for this multi-parameter inversion. Based on the RVoG model, some refined models have also been proposed to adapt to different situations. The S-RVoG model took the terrain conditions into account $[9,10]$ and Gaussian Vertical Backscatter (GVB) model focused on the vertical heterogeneity of the wave-canopy interactions for low frequencies [11]. The inversion error model on polarimetric system parameters were established to correct for systematic bias [12], and the uncertainties in canopy height estimation were estimated in [13]. To reduce computational complexity of RVoG, the three-stage inversion method was proposed and had achieved great effect [14-16]. Nevertheless, the interferometric height precision is easily affected by the data acquisition modes, such as the band, the incidence angle, the baseline length, and the interference pattern (single-pass pattern or repeat-pass pattern) $[3,17]$, which suggests multi-baseline, multi-frequency measurements, and LiDARaided methods can promise more accurate estimations $[18,19]$. Considering this, the fusion of multi-source data becomes a mainstream research $[20,21]$ and some machine learning methods such as support vector machine (SVM) and neural networks (NN) have a great performance in this field [22].

Actually, when the RVoG model is applied to single-baseline repeat-pass PolInSAR data, the uncertainty of temporal decorrelation can significantly reduce the accuracy of height estimation [23]. Howard et al. analyzed multiple decorrelations in radar signals and established a general model of temporal decorrelation [24]. Then Lavalle et al. proposed the RMoG model, improving the estimation of temporal decorrelation by modeling the motion of the scatterers $[25,26]$. An alternative method to compensate for the temporal decorrelation is to scale the volume coherence by a real-valued temporal decorrelation factor, which is adopted by RVoG-vtd model [27]. These two models both increase the number of unknown parameters in RVoG model, making the parameter inversion problem ambiguous. To deal with this problem, two methods are usually adopted, i.e., training certain parameters or solving the global nonlinear least squares problem [27]. However, Supervised training relies on considerable data and rarely achieves a general conclusion. The prior information may also be misleading. The global nonlinear least squares problem with constraints can be hard to solve and lead to higher time and space complexity. Recently, a four-stage inversion algorithm was proposed to decrease the number of unknown parameters in the RVoG-vtd model, which explored the relationship between the mean extinction and the segmentation of the fitted line [28]. Although the height estimation results are much better than three-stage inversion algorithm for RVoG model, it still needs to be further improved. The proposed four-stage algorithm differs from the previous one mainly in the following two aspects: (i) adaptive estimation of the temporal decorrelation in the third stage, and (ii) distance metric transformation in the fourth stage. In this framework, the temporal decorrelation is further modeled and estimated by EM algorithm without the need for any prior information. Moreover, both the amplitude and the phase of volume-only coherence are effectively used for distance metric.

The study area of this paper is Lope National Forest Park, which is one of the four test sites of AfriSAR campaign in Gabon, Africa. The AfriSAR campaign was a joint effort between NASA and ESA which collected both PolInSAR and LiDAR airborne data sets in the tropical areas covering different forest structures, biomass levels and disturbances, to further support the BIOMASS mission which was selected as the seventh Earth Explorer mission with the purpose to estimate forest biomass and height with full coverage over the tropical areas. The PolInSAR and LiDAR data of UAVSAR as well as FSAR were widely used to obtain estimations of forest biomass and height [22,29-31]. However, most of the research focused on the multi-baseline case and the fusion of PolInSAR and LiDAR data. The lack of the assessment for single-baseline case needs to be addressed because of the fundamental role and the low complexity of single-baseline PolInSAR.

This paper focuses on height estimation of single-baseline repeat-pass PolInSAR data. A general model and a novel four-stage inversion algorithm are separately proposed to 
improve accuracy and solve the problem of ambiguity. Section 2 introduces the new model, in which the scatterer distribution and scatterer motion are modeled, respectively. The novel four-stage algorithm with the additive temporal decorrelation estimation stage and the modified height estimation stage is also presented. Section 3 assesses models and algorithms by estimating forest height from the real single-baseline repeat-pass PolInSAR data of Lope National Forest Park in Gabon, Africa, and comparing the results with corresponding LiDAR data. The experiment results indicate the new method has a great potential to compensate for the temporal decorrelation and obtain more accurate inversion results. The discussion and conclusion are given in Sections 4 and 5, respectively.

\section{Model-Based Inversion Algorithm}

\subsection{RVoG Model and Three-Stage Inversion Process \\ 2.1.1. RVoG Model}

With the following three hypotheses: the hypothesis of random volume scattering, the hypothesis of numerous scatterers and the hypothesis of homogeneous distribution of scatterers, Treuhaft et al. proposed the random volume (RV) model defined as follows [7]:

$$
\begin{aligned}
\gamma_{v} & =\frac{\left.p_{1}\left(e^{p_{2} h_{v}}-1\right)\right)}{\left.p_{2}\left(e^{p_{1} h_{v}}-1\right)\right)} \\
p_{1} & =\frac{2 \kappa_{e} \cos \left(\tau_{c}\right)}{\cos \left(\theta-\tau_{c}\right)} \\
p_{2} & =p_{1}+j k_{z}
\end{aligned}
$$

where $k_{z}$ means the vertical wavenumber, which is depended on the wavelength $\lambda$, incidence angle $\theta$ and incidence angle difference $\Delta \theta . \tau_{c}$ is range-facing terrain slope angle, which can be useful in complex terrain case. $h_{v}$ and $\kappa_{e}$ are unknown parameters indicating vegetation height and wave extinction, respectively. The vertical wavenumber $k_{z}$ can be calculated as follows in the bistatic repeat-pass case [27]:

$$
k_{z}=\frac{4 \pi \Delta \theta \cos \left(\tau_{c}\right)}{\lambda \sin \left(\theta-\tau_{c}\right)}
$$

Based on the RV model, Treuhaft et al. modeled the ground scattering and the interaction between ground and trunk in the forest area, and further obtained the RVoG model, which is widely used in the field of forest height inversion. Its relationship with the RV model can be expressed as [8]:

$$
\gamma(\omega)=e^{j \phi_{0}} \frac{\mu(\omega)+\gamma_{v}}{\mu(\omega)+1}
$$

where $\phi_{0}$ denotes the interferometric ground phase and different $\omega$ represents different polarization modes. $\mu(\omega)$ is the ground-to-volume backscattering ratio, which lies in the range $0 \leq \mu(\omega) \leq \infty$ in the ideal case, with limits representing pure surface scattering $(\mu(\omega)=\infty)$ and pure volume scattering $(\mu(\omega)=0)$. The increase of unknown parameters in the RVoG model makes the nonlinear inversion process more complicated.

\subsubsection{Three-Stage Inversion Process}

The three-stage inversion method proposed by Cloude et al. [14] is based on the RVoG model and greatly reduces the complexity of the inversion procedure. Therefore, it has been commonly used and has achieved great effect in many cases. The characteristic that the coherence under different polarization states are distributed on a straight line in the complex unit circle (CUC) has been used effectively to obtain the ground phase $\phi_{0}$ and volume coherence $\gamma_{v}$. The three-stage inversion process can be conducted as follows:

- $\quad$ Least squares line fit. Since Equation (5) indicates that coherence values in different polarization states lie along a straight line in CUC, the first stage is to find the best-fit 
line of interferometric coherence values in different polarization modes, such as $\mathrm{HH}$, $\mathrm{VV}, \mathrm{HH}-\mathrm{VV}, \mathrm{HH}+\mathrm{VV}$, and HV.

- Ground phase removal. In the second stage, ground phase must be determined and removed from the coherence. The phases of two intersection points of the straight line and the CUC are the candidates of ground phase. Generally, the relative location of coherence values in different polarization states along the best-fit line arranges according to Figure 1, which becomes one criterion for distinguishing the real ground phase.

- Height and extinction estimation. The pre-calculate look up table (LUT) of volumeonly coherence is employed to estimate vegetation height and mean extinction in last stage. The parameters are determined by minimizing the distance between the calculated volume coherences and the observed volume coherence.

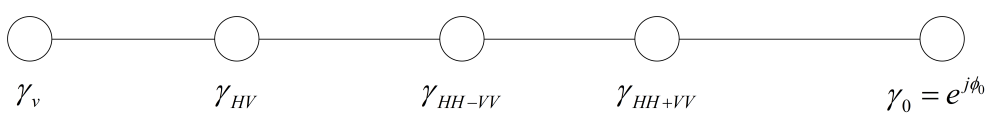

Figure 1. The relative location of coherence values in different polarization states along the bestfit line.

\subsection{RVoG-vtd Model and Four-Stage Inversion Algorithm}

\subsubsection{RVoG-vtd Model}

The temporal decorrelation is a major source of decorrelation especially under the repeat-pass case. When the RVoG model is adapted in repeat-pass case, dealing with temporal decorrelation becomes an essential step. The RVoG-vtd model proposes the idea of compensating for the effect of temporal decorrelation by adding a real-valued factor. Then, the model becomes:

$$
\gamma_{v t d}(\omega)=e^{j \phi_{0}} \frac{\mu(\omega)+\alpha_{v t} \gamma_{v}}{\mu(\omega)+1}
$$

where $\alpha_{v t}$ is the temporal decorrelation factor applied to the volume coherence. The rest of the RVoG model equations are unaffected.

\subsubsection{Four-Stage Inversion Algorithm}

Due to the addition of parameter, the solution is now ambiguous for single-baseline data. In contrast to the methods such as supervised training and solving the global nonlinear least squares problem mentioned above, a four-stage inversion algorithm is adopted to solve the ambiguous problem and keep the calculation complexity at a low level simultaneously. The volume scattering phase center moves to the top of the canopy according to the higher mean extinction value, which indicates the relative position of the observed volumetric coherence on the coherence line can be employed to limit the range of mean extinction coefficient [15]. In this framework, an index was suggested to interpret the relative location of the observed volume coherence, $\gamma_{H V}$, on the coherence line as [15]:

$$
D . I=\frac{A . L}{V . L}
$$

wherein D.I is the distance ratio index of the ambiguous line length (A.L.) and the visible line length (V.L.), as shown in Figure 2. With the expectation that the mean extinction value and D.I. value is inversely related, Managhebi et al. defined the mean extinction coefficient as the following linear function [28]:

$$
\kappa_{e}=a D . I+b
$$

where $\kappa_{e}$ is the mean extinction coefficient, D.I is the distance ratio index, $a$ and $b$ are the model parameters computed by least squares method using real L-band PolInSAR data pair. 


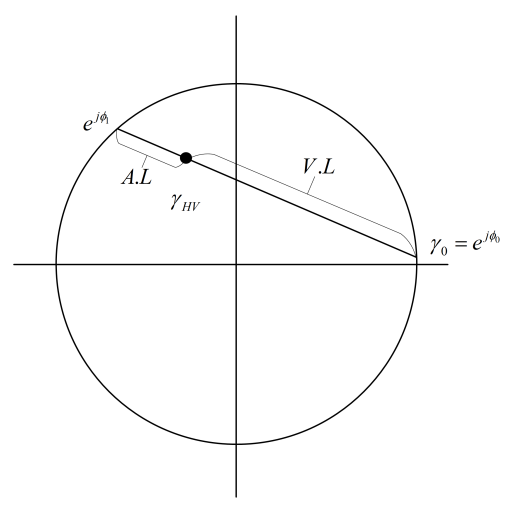

Figure 2. The ambiguous line segment and the visible line segment on the fitted line.

To summarize, the four-stage inversion process consists of the following four steps:

- $\quad$ Least squares line fit.

- Ground phase removal.

- Extinction estimation.

- Volume height and temporal decorrelation estimation.

The first two steps are similar to the three-stage inversion process. In the third step, D.I and the mean extinction coefficient are calculated using Equations (7) and (8). Then the fixed mean extinction coherence locus is determined to estimate the volume height and the real temporal decorrelation multiplying factor from the intersection point between the volume coherence loci and $\phi=\phi_{v}$.

\subsection{GRVoG-vtd Model and a Novel Four-Stage Inversion Algorithm \\ 2.3.1. GRVoG-vtd Model}

Since more parameters make it hard to achieve a unique and precise solution, a direct idea is to set wave extinction to a reasonable value for reducing variable. However, in the RVoG model, it is assumed that the scatterer density of the forest from the surface to the canopy is a constant, which can simplify the derivation process and the model function. Therefore, the wave extinction values in the model are not necessarily indicative of the actual extinction. In practice, the extinction parameter in the RVoG model is sensitive to changes in the vertical structure [27], which means before fixing wave extinction, the forest structure should be modeled carefully. Presently, the LiDAR data can provide a profile of scatterer vertical distribution of forest [18], which varies from species to species. Therefore, in this research, the generalized RVoG (GRVoG) model with general scatterer vertical distribution is first introduced.

Start by the distribution of volume scatterer density $\rho(z)$, the volume coherence in GRVoG model can be derived in Appendix A as [7]:

$$
\gamma_{v}^{G}=\frac{\int_{0}^{h_{v}} \exp \left(j k_{z} z\right) \rho(z) \exp \left(\frac{\left.-2 \kappa_{e} \int_{z}^{h_{v}} \rho(h) d h\right)}{\cos \theta}\right) d z}{\int_{0}^{h_{v}} \rho(z) \exp \left(\frac{\left.-2 \kappa_{e} \int_{z}^{h_{v}} \rho(h) d h\right)}{\cos \theta}\right) d z}
$$

$\rho(z)$ can reflect the vertical structure of the vegetation. The model degenerates to RVoG when $\rho(z)$ is set to a constant value. In practice, $\rho(z)$ could vary with the species of trees, crown depth and other physical conditions.

The GRVoG-vtd model compensates for temporal decorrelation with a real-value factor similarly to the RVoG-vtd model as follows:

$$
\gamma_{v t d}^{G}(\omega)=e^{j \phi_{0}} \frac{\mu(\omega)+\alpha_{v t}^{G} \gamma_{v}^{G}}{\mu(\omega)+1}
$$


To solve the ambiguous problem caused by adding parameter $\alpha_{v t}^{G}$, this paper also explores the relationship between the temporal decorrelation and forest height. The temporal decorrelation follows from physical changes and is closely related to the motion of scatterers in a general view. Assuming Gaussian-statistic random motion, like [25], the temporal decorrelation can be denoted in following form:

$$
\alpha_{v t}^{G}\left(h_{v}\right)=\frac{\int_{0}^{h_{v}} \rho(z) \exp \left(-\frac{1}{2}\left(\frac{4 \pi}{\lambda}\right)^{2} \sigma_{r}^{2}(z)\right) d z}{\int_{0}^{h_{v}} \rho(z) d z}
$$

where $\sigma_{r}(z)$ means the standard deviation of Gaussian distribution in the layer of height $\mathrm{z}$. To derive an explicit expression for $\alpha_{v t}^{G}\left(h_{v}\right), \rho(z)$ in this model is set to be a constant value $\rho_{0}$ like RVoG model and a first-order approximation of the motion variance is considered [26]:

$$
\sigma_{r}^{2}(z)=\sigma_{g}^{2}+\beta_{0} z
$$

with $\sigma_{g}^{2}$ represents the motion variance of scatterers on the ground. Since $\sigma_{g}^{2}$ is independent of height, it can be moved out of integration and estimated as a constant $\alpha_{g}$. The GRVoG-vtd model can be derived as:

$$
\begin{aligned}
\gamma_{v t d}^{G}(\omega) & =e^{j \phi_{0}} \frac{\mu(\omega)+\alpha_{v t}^{G}\left(h_{v}\right) \gamma_{v}^{G}\left(h_{v}, \kappa_{e}\right)}{\mu(\omega)+1} \\
\alpha_{v t}^{G}\left(h_{v}\right) & =\frac{\alpha_{g} \int_{0}^{h_{v}} \exp (-\beta z) d z}{h_{v}}
\end{aligned}
$$

with $\beta=-\frac{1}{2}\left(\frac{4 \pi}{\lambda}\right)^{2} \beta_{0}$ is a constant corresponding to the assumption of $\sigma_{r}^{2}(z)$ which can be empirically determined. It can be seen that GRVoG-vtd degenerates to RVoG-vtd when $\beta_{0}=0$ and $\rho(z)$ is a constant function.

\subsubsection{A Novel Four-Stage Inversion Algorithm}

Based on the GRVoG-vtd model, this paper proposed a novel four-stage inversion algorithm. The unknown parameters in GRVoG-vtd are the vegetation height $h_{v}$, the mean extinction coefficient $\kappa_{e}$, and the constant real-value decorrelation factor $\alpha_{g}$. According to the form of the GRVoG-vtd model, one can see that the coherences in different polarization states are still distributed on a straight line in the CUC with an intersection point $e^{j \phi_{0}}$. Therefore, the first two stages of the method are still the least squares line fit and removal of the ground phase similarly to the three-stage inversion process. The volume coherence obtained after the first two steps actually includes the temporal decorrelation factor and can be expressed as $\alpha_{v t}^{G}\left(h_{v}\right) \gamma_{v}^{G}\left(h_{v}, \kappa_{e}\right)$. Since the sparse savannas and the dense forests in vegetation area show a clear difference in volume coherence, the third stage uses EM algorithm to classify these species and estimate the constant factor $\alpha_{g}$, simultaneously.

Assuming the distribution of volume coherence amplitude $(\rho)$ is a Gaussian mixture model (GMM) with $K$ components, the probability of the coherence amplitude is:

$$
p(\rho)=\sum_{k=1}^{K} \pi_{k} \mathcal{N}\left(\rho \mid \mu_{k}, \sigma_{k}^{2}\right)
$$

where $\mu_{k}, \sigma_{k}^{2}$ represent the mean value and the variance of the k-th Gaussian distribution, respectively. $\pi_{k}$ is the proportion of the $\mathrm{k}$-th component. Let $l_{n k}$ denote the probability that the $\mathrm{n}$-th sample $\rho_{n}$ belongs to the k-th components. The EM algorithm adopts alternate iterations of the Expectation step and the Maximization step until convergence to divide the samples into corresponding component without any prior information. 
E-step:

$$
l_{n k}=\frac{\pi_{k} \mathcal{N}\left(\rho_{n} \mid \mu_{k}, \sigma_{k}^{2}\right)}{\sum_{k=1}^{K} \pi_{k} \mathcal{N}\left(\rho_{n} \mid \mu_{k}, \sigma_{k}^{2}\right)}
$$

M-step:

$$
\begin{aligned}
& \mu_{k}^{\text {new }}=\frac{1}{N_{k}} \sum_{n=1}^{N} l_{n k} \rho_{n} \\
& \sigma_{k}^{2^{\text {new }}}=\frac{1}{N_{k}} \sum_{n=1}^{N} l_{n k}\left(\rho_{n}-\mu_{k}^{\text {new }}\right)^{2} \\
& \pi_{k}^{\text {new }}=\frac{N_{k}}{N}
\end{aligned}
$$

where

$$
N_{k}=\sum_{n=1}^{N} l_{n k}
$$

Since the height of sparse savannas mainly distributes under $3 \mathrm{~m}$, the volume decorrelation is inconspicuous compared to temporal decorrelation. Accordingly, the amplitude of coherence in savanna region reflects the real-value factor $\alpha_{g}$. Let the k-th component represents sparse savannas, then

$$
\hat{\alpha}_{g}=\frac{\sum_{n=1}^{N} l_{n k} \rho_{n}}{N_{k}}
$$

The fourth stage is to estimate the vegetation height and extinction coefficient. The LUT of vegetation height, extinction coefficient, and volume coherence can be pre-calculated similarly to three-stage inversion algorithm. The main difference is the generalized distance is used to replace the Euclidean distance in the original algorithm. The shortest Euclidean distance criterion in the original algorithm does not consider the importance and reliability of coherence amplitude and phase, which would significantly reduce the precision of inversion since the distribution of volume coherence is inhomogeneity in practice. This paper proposes a generalized distance to measure the similarity of amplitude and phase respectively and obtain a more reasonable inversion result.

$$
d_{i j}=\sqrt{\lambda\left(\rho_{i}-\rho_{j}\right)^{2}+(1-\lambda)\left(\phi_{i}-\phi_{j}\right)^{2}}
$$

where $\rho_{i}, \phi_{i}$ are the amplitude and the phase of the i-th coherence. For sparse savanna region obtained in the third stage, the coherence amplitude is large, and the distribution is concentrated, which indicates the height inversion result mainly depends on the phase of the volume coherence. Therefore, in the generalized distance the parameter $\lambda$ is relatively small for this type. On the contrary, the coherence amplitude is small for dense forest and the uncertainty of phase can cause more error. In this regard, the coherence amplitude should occupy a larger proportion in the generalized distance measurement.

In summary, the following step-by-step outline describes the whole process.

1. Generate the coherence in different polarization states and fit the least square line in the CUC.

2. Choose the ground underlying phase from the two intersection points between the best fitted line and CUC. Calculate the volume coherence by removing the ground phase and projecting the farthest coherence from the ground coherence point to the fitted line. 
3. Classify sparse savannas and dense forest by the amplitude of the volume coherence using EM algorithm. Determine the constant parameter $\alpha_{g}$ by calculating the mean value of the amplitude in sparse savanna region.

4. Estimate the vegetation height and mean extinction based on the pre-calculate LUT of GRVoG-vtd model by minimizing the generalized distance between calculated volume coherences and the observed volume coherence.

Figure 3 shows the flowchart of proposed four-stage algorithm. The pre-processing and the first two stages are carried similarly to the three-stage algorithm. The main difference is the addition of the third stage including vegetation classification and constant factor estimation as well as the transformation of the distance form from the Euclidean distance to the generalized distance. The extinction coefficient is determined by the length of the segment line in the previous four-stage algorithm for RVoG-vtd model. Then the coherence curve is selected by fixed extinction and the vegetation height is estimated based on the phase of the coherence. In the novel four-stage algorithm for GRVoG, the order to estimate parameters has changed. The temporal decorrelation factor is estimated first and the coherence amplitude and phase are both used to get more precise vegetation height. In the generalized distance, the ratio $\lambda$ is set to assign weights of amplitude and phase to reflect the reliability and importance of the two factors for the purpose of adapting to different situations.

\subsection{Analysis of Models and Corresponding Algorithms}

The differences of different models, i.e., RVoG, RVoG-vtd, and GRVoG-vtd are visually illustrated in Figure 4. For mean extinction coefficient $\kappa_{e}$ varying from 0 to 0.9 , the LUTs of RVoG, RVoG-vtd with a fixed $\alpha_{v t}$ and, GRVoG-vtd with a fixed $\alpha_{g}$ are scattered on CUC, respectively. Different colors represent different mean extinction values, and the curve becomes more concentrated as the decrease of the mean extinction. Under the ideal case, the coherence locates at the point $(1,0)$ when the vegetation height is 0 . Consequently, the curve of RVoG begins from the point $(1,0)$ and gradually shrink into the center of CUC for the influence of temporal decorrelation factors is ignored. Correspondingly, the curves of RVoG-vtd and GRVoG-vtd start from the points $\left(\alpha_{v t}, 0\right)$ and $\left(\alpha_{g}, 0\right)$ respectively. The RVoG-vtd model provides a scale factor for the whole table, which has not changed the shape of the curve. In contrast, the addition of the temporal decorrelation factor in the GRVoG-vtd model makes the curve converge rapidly and the correspondence between coherence and vegetation height has changed significantly.

As Figure 4 demonstrates, the LUTs of the three models appear as high curvature curves in the CUC, which cause great difficulties in subsequent estimation. However, the curve has a potential to be manifold embedded. Taking the LUT of GRVoG-vtd as an example, Figure 5 explores whether the amplitude-phase plane can better represent the LUT. Compared to the CUC plane, the curve in the amplitude-phase plane shows more linear characteristics and can be easier distinguished, which suggests that the distance in the amplitude-phase plane is more efficient to measure the similarity and estimate vegetation heights. To further explain this statement and seek for a more reasonable criterion, several basic approaches to estimate vegetation height are conducted and analyzed in Figures 6 and 7. 


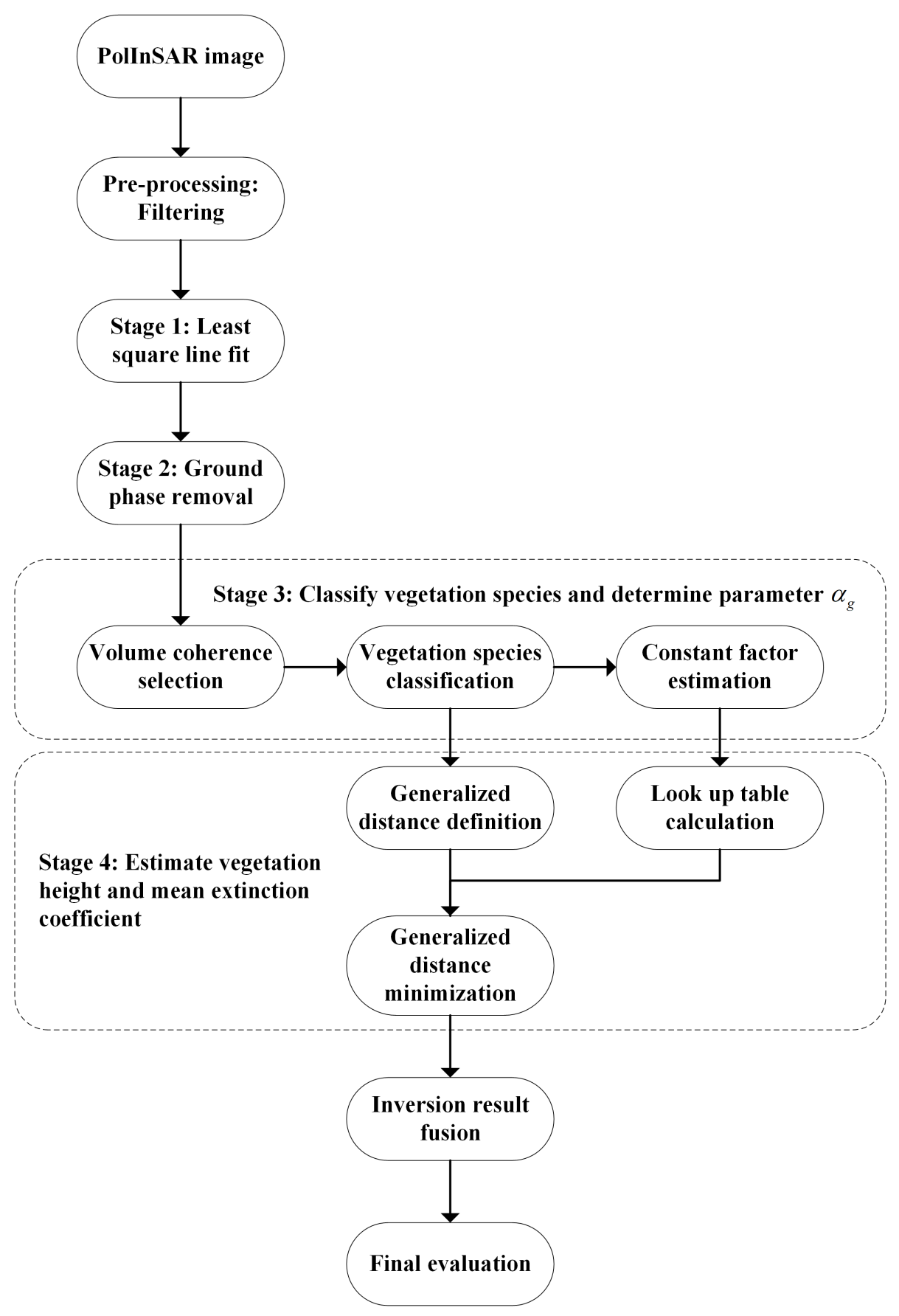

Figure 3. The flowchart of the novel four-stage algorithm.
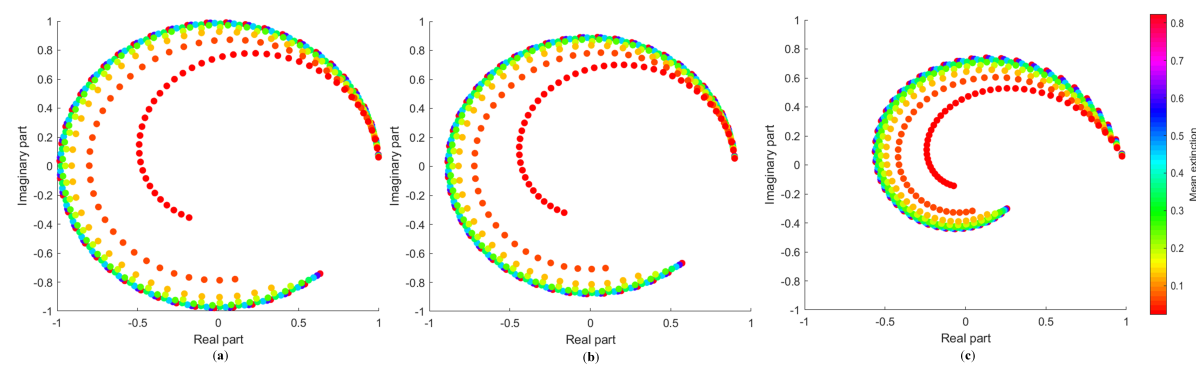

Figure 4. The LUTs of (a) RVoG model, (b) RVoG-vtd model (fixed $\alpha_{v t}$ ) and (c) GRVoG-vtd model (fixed $\alpha_{g}$ ) with different values of mean extinction coefficient $\kappa_{e}$. 


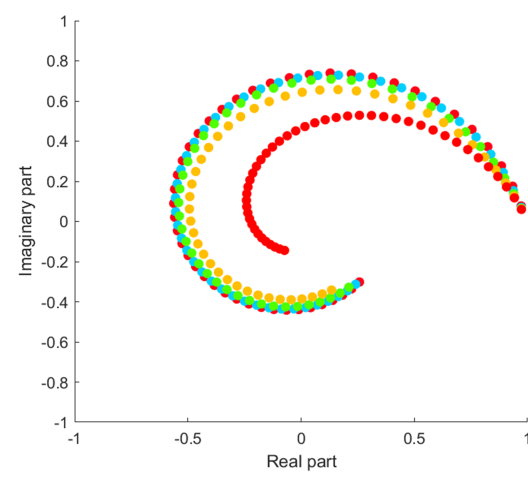

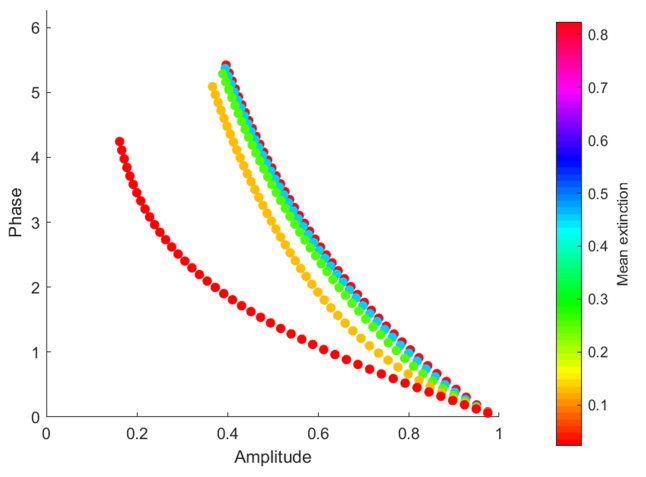

Figure 5. The LUTs in different planes: (a) the CUC plane and (b) the amplitude-phase plane.

Figure 6 shows the relationship between the regions in the CUC plane and the vegetation heights based on the Euclidean distance, the amplitude distance and the phase distance respectively defined as follows:

$$
\begin{aligned}
& d_{i j}^{\text {Euc }}=\sqrt{\left(x_{i}-x_{j}\right)^{2}+\left(y_{i}-y_{j}\right)^{2}} \\
& d_{i j}^{a m p}=\left|\rho_{i}-\rho_{j}\right| \\
& d_{i j}^{p h a}=\left|\phi_{i}-\phi_{j}\right|
\end{aligned}
$$

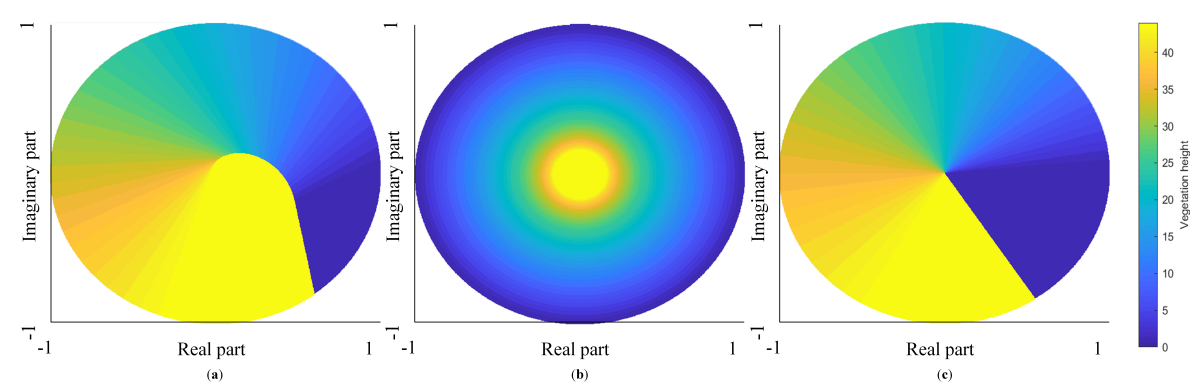

Figure 6. The mapping of vegetation height and coherence in the CUC plane using least distance criterion with (a) the Euclidean distance, (b) the amplitude distance and (c) the phase distance.

In practice, using these three distances is not enough to truly reflect the similarity between observed coherence and model-based coherence for effective estimation of vegetation height. Since the height discrimination is clearer in the amplitude-phase plane as discussed above, we consider solving the estimation problem in this new plane. The corresponding regions in the amplitude-phase plane for these three distances are also given in Figure 7a-c diagrams, respectively. It can be seen that the least distance criterion in CUC lead to an unexpected classification region which has poor interpretability referring to the ideal curve and the amplitude of coherence plays an insignificant role under most conditions. In this regard, the generalized distance is proposed to measure the similarity more equitably for the amplitude and the phase. The typical corresponding regions are shown in the diagram of Figure $7 \mathrm{~d}$ which has stronger consistency with the calculated curve. Nevertheless, the amplitude and phase are affected by varying degrees of noise which should be considered carefully to reach higher accuracy. Consequently, the parameter $\lambda$ is set according to the situations. 

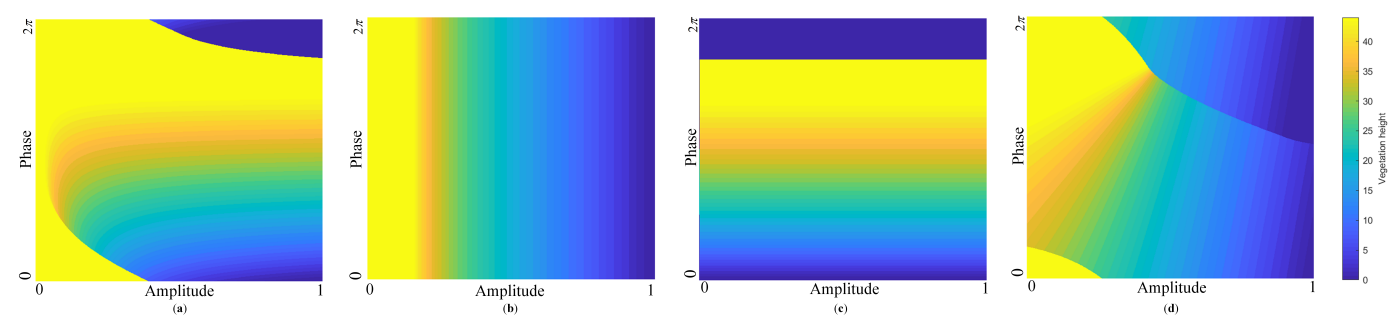

Figure 7. The mapping of vegetation height and coherence in the amplitude-phase plane using least distance criterion with (a) the Euclidean distance, (b) the amplitude distance, (c) the phase distance and (d) the generalized distance.

\section{Results}

\subsection{Study Area}

The study area is the Gabon Lope Park region which locates on the west coast of Africa with the loci of $0^{\circ} 30^{\prime} 00^{\prime \prime} \mathrm{S} 11^{\circ} 30^{\prime} 00^{\prime \prime} \mathrm{E}$ and an area of $4910 \mathrm{~km}^{2}$ as Figure 8 shows. It covers diverse habitats for its over 70 years history of being a wildlife reserve and therefore is an ideal test site to estimate forest biomass and height. Here, the focus is on a small area of interest in the northeastern Lope with the central longitude $11^{\circ} 34^{\prime} 00^{\prime \prime} \mathrm{E}$ and latitude $0^{\circ} 13^{\prime} 30^{\prime \prime} \mathrm{S}$.

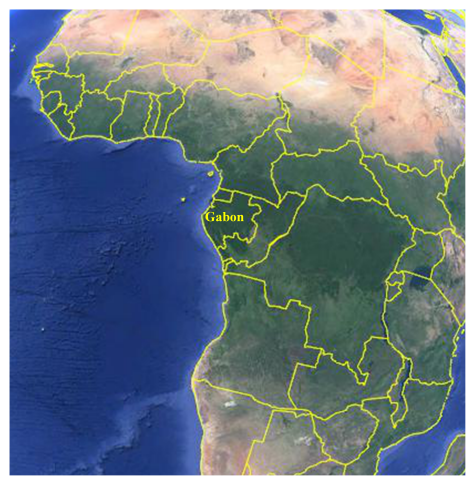

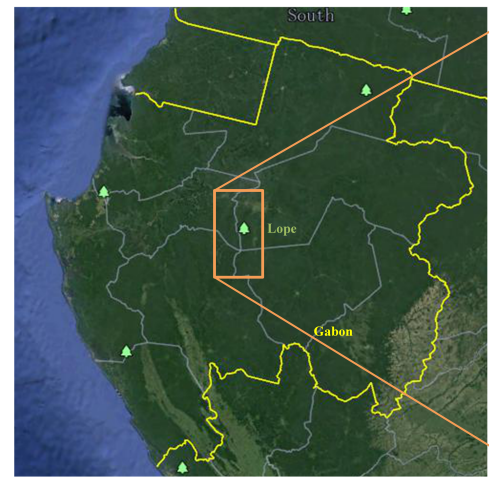

(b)

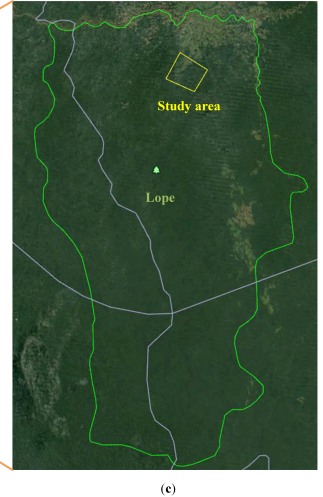

Figure 8. The location of experimental sites. (a) Gabon, (b) Lope National Park, (c) Study area (Obtained in the Google Earth).

\subsection{Data Set}

The experimental data consists of the airborne single-baseline repeat-pass PolInSAR data and corresponding LiDAR data in Lope National Park region.

The PolInSAR data was obtained by the 2-nd pass and the 4-th pass of the 11-th flight in February2016 in the AfriSAR campaign by FSAR of the German Aerospace Center (DLR). The frequency band is L-band, and the central frequency is $1.3 \mathrm{GHz}$. The range and azimuth resolution of this SAR data are $1.92 \mathrm{~m}$ and $0.65 \mathrm{~m}$, respectively. The campaign introduction and data can be found in the European Space Agency (ESA) website (https: / / earth.esa.int/ eogateway / campaigns/afrisar-2016).

The LiDAR data used in this study was collected by Land Vegetation and Ice Sensor (LVIS). As in [10,18,22], the LVIS RH100 metrics were chosen to validate the PolInSAR forest height estimations. The LiDAR-based vegetation height has been compared to TanDEM-X DEM in AfriSAR Final Report [32] which found that the top of the canopy computed from the LiDAR data is extremely close to the TanDEM-X data as expected. The LiDAR-based height also has a strong consistency with the ground data in different plots which proved its strong reliability in the previous work [22]. Therefore, in this work, we decided to compare height estimations of PolInSAR via different algorithms with LVIS RH100 metrics to verify their performance.

Although the height precision is high for LiDAR measurements, the horizontal resolution of LVIS is much lower, which is about 25 m, indicating that the LVIS RH100 metrics 
need to be interpolated to fit PolInSAR geometry. Consequently, the PolInSAR image show more speckle characteristics and there will be a large deviation when using a single pixel to do height estimation. Therefore, this paper uses $50 \times 50$ pixels as a block in the subsequent quantitative analysis, which is more reasonable considering LiDAR resolution.

In this data set, the overlapped region of PolInSAR and LiDAR covers a size of about $7000 \times 1000$ pixels in the PolInSAR image. However, there are some small pieces with missing values. We intercept an interested area with the size of $3000 \times 1000$ pixels from the whole image which contains few missing values and large fluctuations. The Pauli image and LVIS RH100 height image of the interested area are showed in Figure 9, which consists of sparse savannas and dense forests of varying heights up to $60 \mathrm{~m}$.

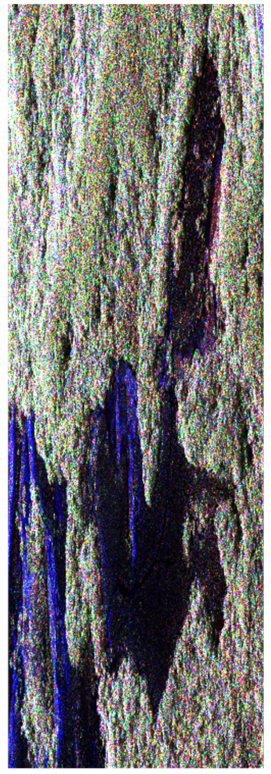

(a)

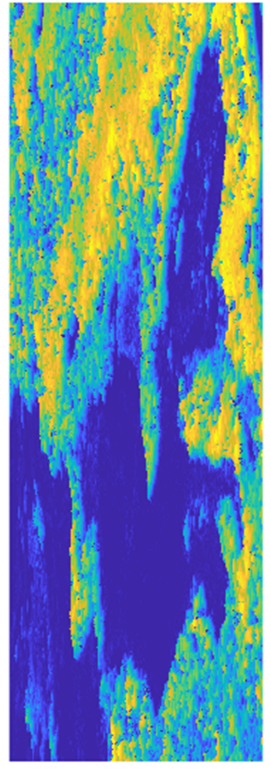

(b)

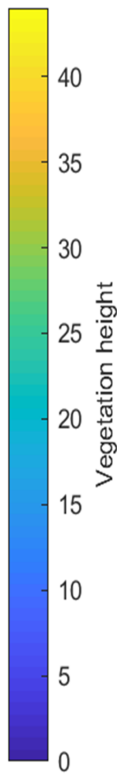

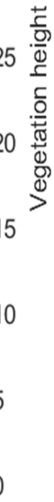

Figure 9. (a) The Pauli image and (b) the LVIS RH100 height image of the interested region.

\subsection{Experimental Results}

The observed coherences attained in the first two stages can be clearly divided into two vegetation species for the coherence amplitude shows a distinct bimodal distribution as demonstrated in Figure 10. The two-component GMM is reasonably assumed and EM algorithm is adopted to adaptively distinguish these categories without any prior information.

The calculated coherences represented by the curve in Figure 5 illustrates the LUT in the amplitude-phase plane is closer to straight lines. To verify this statement, the observed coherences sampled at the rate of $0.02 \%$ with varying heights are scattered in the CUC plane and the amplitude-phase plane respectively as Figure 11 shows. As expected, the distribution of coherence in the amplitude-phase plane is obviously more linear, which brings great convenience to the subsequent height discrimination. However, we can also see that the coherence is greatly affected by the noise, which indicates the block of $50 \times 50$ pixels is necessary. 


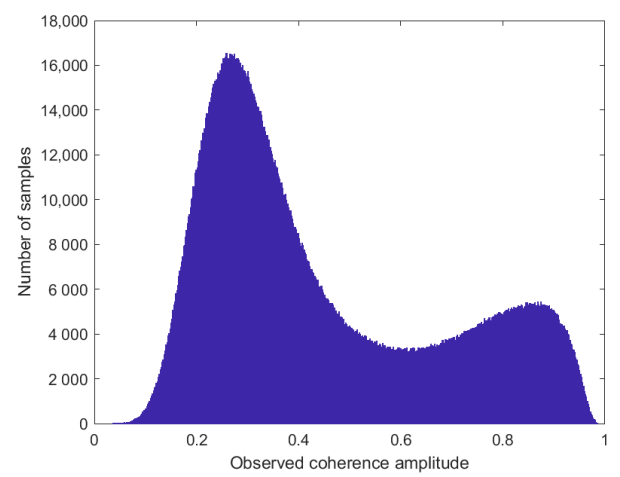

Figure 10. The distribution of observed coherence amplitude.

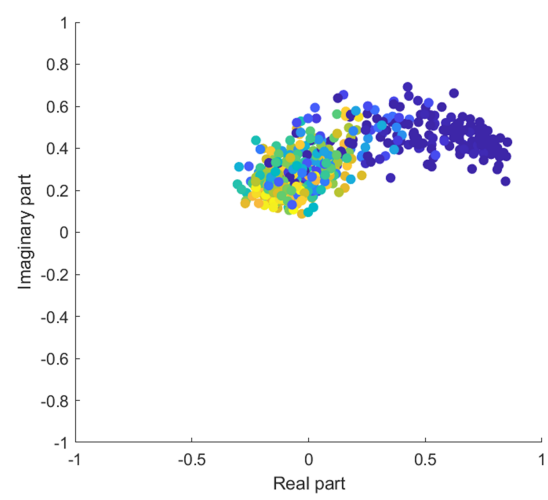

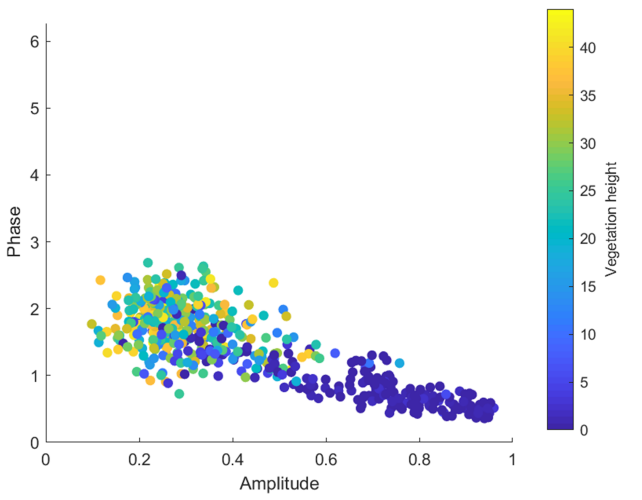

(b)

Figure 11. The distribution of observed coherence in the (a) CUC plane and (b) amplitudephase plane.

The visualized height estimation results of these three models are all shown in Figure 12. The inversion results show the characteristics of each algorithm in a macroscopic way. The RVoG model does not compensate for the temporal decorrelation, which results in the high calculated coherence amplitude. Nevertheless, due to the influence of temporal decorrelation, the observed coherence amplitude is low, which makes the algorithm overestimate in most areas. After the estimation of the extinction coefficient, the height is only relative to the phase in RVoG-vtd model. The lack of the information of coherence amplitude leads to decreased accuracy in areas with high vegetation. The GRVoG-vtd model further models the temporal decorrelation and distinguishes the vegetation types. The temporal decorrelation factor is estimated by regions with low volume decorrelation adaptively and the coherence amplitude and phase are comprehensively used. Therefore, the estimated height has stronger consistency with the corresponding LVIS RH100 height. Still, there are some areas with large estimation errors because it is difficult to accurately estimate the vegetation with large range of height distribution only by using single-baseline data. The accuracy of the three models all depends on $k_{z}$ to some extent. For the area with large $k_{z}$, the maximum value of inversion height is limited due to the wrapped phase, which cannot accurately reflect the tree height. From the view of detail, since the LiDAR image has been interpolated, it is relatively smooth and uniform, the PolInSAR-based results show more speckle characteristics because of the susceptibility to noise and the random fluctuations of the forest. 


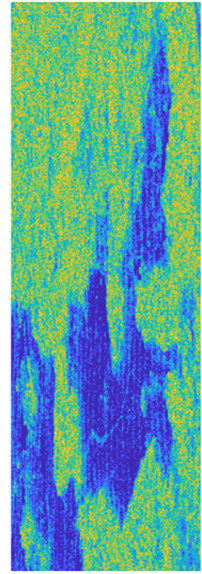

(a)

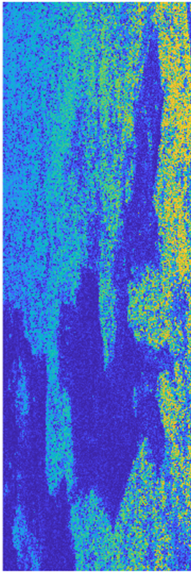

(b)

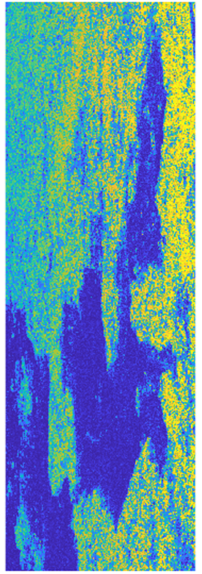

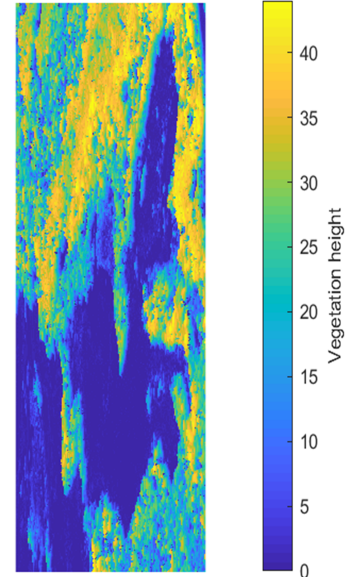

Figure 12. The vegetation height results of (a) the RVoG model, (b) the RVoG-vtd model, (c) the GRVoG-vtd model, and (d) LiDAR measurements.

To obtain intuitive results, we selected three vertical lines distributed in the left, middle and right parts of the image respectively as Figure 13 shows. Each line contains 60 consecutive blocks with significant fluctuations. It can be seen that RVoG overestimates tree height in most areas, especially in low vegetation height area. In contrast, the RVoGvtd model generally underestimates vegetation height because of the lack of amplitude information. The GRVoG-vtd model maintains strong consistency with the LiDAR-based height in a large range of variation and has a stronger adaptability.
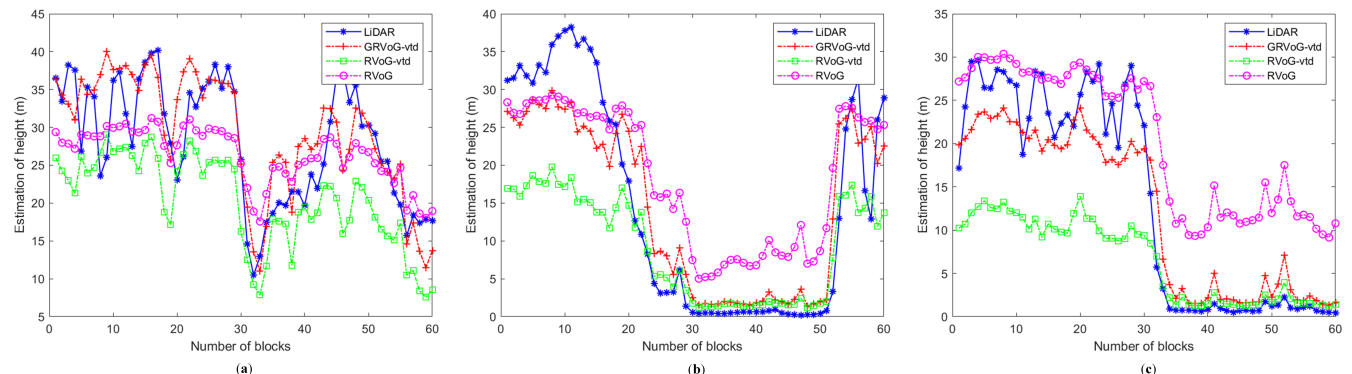

Figure 13. The line views of the estimation of vegetation heights. $(\mathbf{a}-\mathbf{c})$ are three vertical lines distributed in the left, middle and right parts of the image with the column number 25, 500 and 975 , respectively.

A more detailed quantitative analysis is based on dividing the whole map into several small pieces. The whole image is divided into 1200 pieces and each piece has a size of $50 \times 50$ pixels. The height estimation results of RVoG, RVoG-vtd and GRVoG-vtd are assessed versus LiDAR height, respectively. As shown in Figure 14, each point represents one piece of the image. The scatter plot also illustrates that the RVoG model overestimates most of the block regions. The GRVoG-vtd model and RVoG-vtd model both have a great potential to effectively compensate for the effect of temporal decorrelation and have a more reasonable output. Compared to RVoG-vtd, GRVoG-vtd is more elaborate and can take full advantage of the amplitude and phase of volume coherence, hence the heights estimated are more reliable. Since $k_{z}$ has a significant influence on the output for single-baseline data [33], the phase will be wrapped when tree height is too high, hence the errors of inversion results will be larger for vegetations with height over $30 \mathrm{~m}$. 

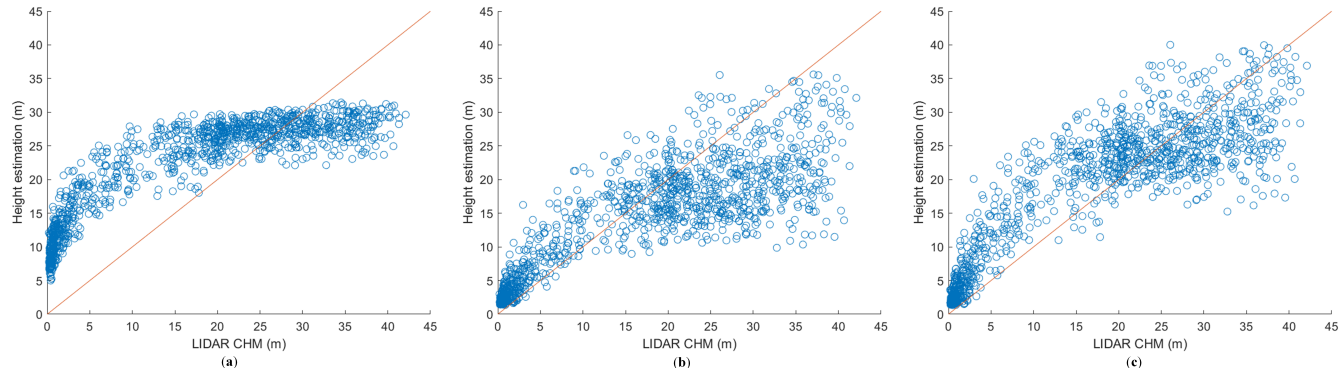

Figure 14. Validation of the inversion results of (a) the RVoG model, (b) the RVoG-vtd model and (c) the GRVoG-vtd model versus LiDAR-based height.

The quantitative comparison of the employed methods with respect to LiDAR measurements are listed in Table 1, which include bias, RMSE and $R^{2}$. As expected, the RVoG model has an overestimation of $4.81 \mathrm{~m}$ averagely and its RMSE is the highest. Both RVoGvtd and GRVoG-vtd have a significant improvement compared to RVoG. From the three indicators, we can also see the superiority of the GRVoG-vtd model in this experiment.

Table 1. Quantitative comparisons of the employed methods with respect to LiDAR measurements.

\begin{tabular}{cccc}
\hline Model & Bias & RMSE & $\boldsymbol{R}^{\mathbf{2}}$ \\
\hline RVoG & 4.8123 & 8.6904 & 0.8699 \\
RVoG-vtd & -2.8665 & 7.7168 & 0.8438 \\
GRVoG-vtd & 1.2764 & 6.2341 & 0.8783 \\
\hline
\end{tabular}

\section{Discussion}

This section further analyzes the inversion errors of different algorithms, and discusses their performances on different vegetation types.

\subsection{Analysis of Inversion Error}

As can be seen from Table 1, the proposed four-stage inversion method for GRVoGvtd model has stronger consistency with LiDAR-based data. To more intuitively reflect the errors of each algorithm, the error distribution functions are given in Figure 15. The expectation values of the error distribution functions are the biases in Table 1. Combined with the scatter diagram of the inversion results in Figure 14, it can be indicated from the error distribution curves that most of the estimation errors of RVoG are distributed on the positive axis for the influence of temporal decorrelation. Due to the lack of effective application for coherence amplitude, more errors of RVoG-vtd are distributed in the negative axis. The overall error distribution of the GRVoG-vtd model proposed in this paper is more concentrated. The standard deviation of the distribution is $\sigma=2.67 \mathrm{~m}$, which is slightly lower than that of the first two methods, which are $2.69 \mathrm{~m}$ and $2.68 \mathrm{~m}$, respectively. The expectation value $\mu=1.28 \mathrm{~m}$, which is also closer to 0 simultaneously. The errors distributed on positive and negative semiaxes are relatively balanced, and most of the errors are distributed in $\mu \pm 3 \sigma$ range, which is $1.28 \mathrm{~m} \pm 7.9 \mathrm{~m}$. Therefore, a better vegetation estimation result can be obtained. 


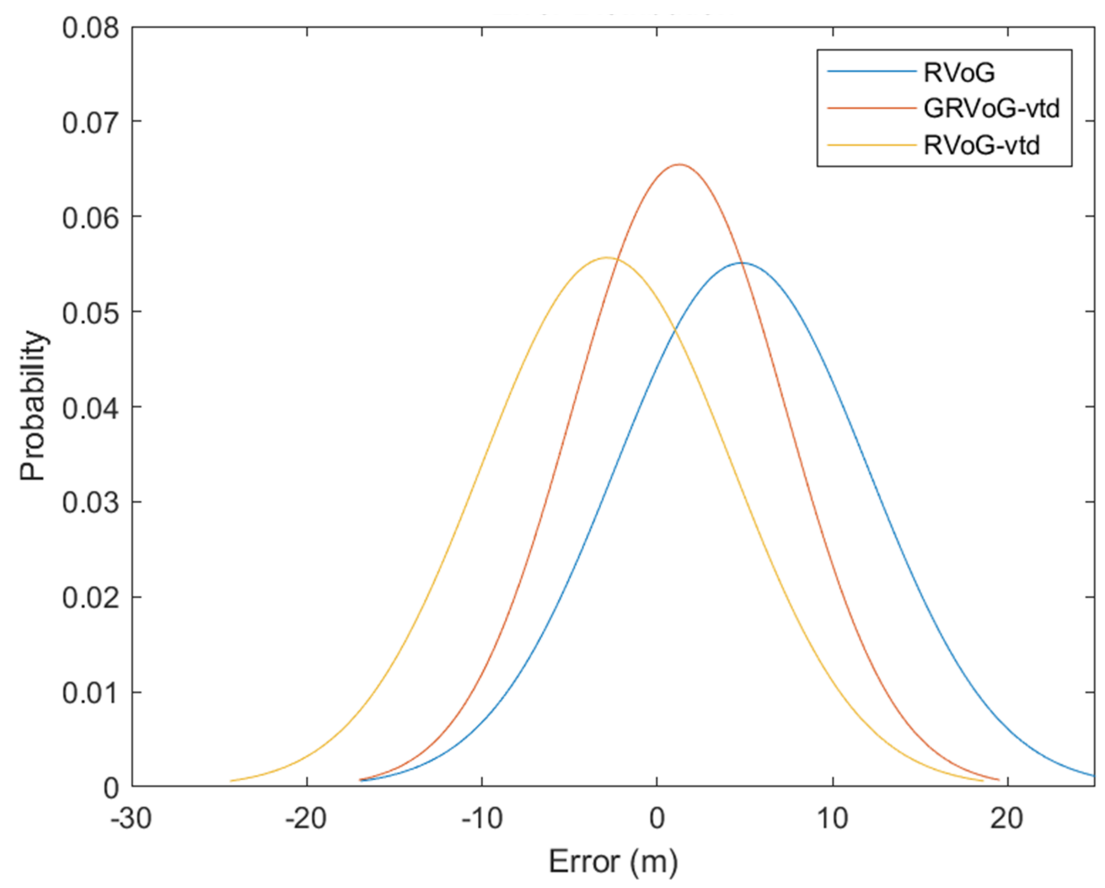

Figure 15. The error distributions of employed algorithms compared to LVIS RH100 heights.

Table 2 divides the vegetation height into three intervals, which represent the sparse savanna $(0-15 \mathrm{~m})$, low forest $(15-25 \mathrm{~m})$, and high forest $(25-60 \mathrm{~m})$ respectively. It can be seen from the table that the RVoG-vtd model performs best in the region with vegetation less than $15 \mathrm{~m}$, followed by the GRVoG-vtd model. The RVoG model performs better in the region of 15-25 m vegetation and GRVoG-vtd is slightly lower than RVoG. In dense forest region, the GRVoG-vtd model shows a better performance due to the adaptive classification of vegetation types and estimation of temporal decorrelation. The proposed method shows high stability in the overall three intervals, and the error distribution is relatively concentrated, which also reflects the conclusion of Figure 15. It can be indicated from Table 2 that for low vegetation, the coherence phase is more effective, while for dense forest, the coherence amplitude should be emphasized to obtain more reasonable results, which shows the rationality of the proposed four-stage inversion algorithm in this paper.

Table 2. Hight estimation error comparisons of three models for different vegetation types. The bold numbers mean the least RMSE and least bias in each row.

\begin{tabular}{ccccccc}
\hline \multirow{2}{*}{ Model } & \multicolumn{2}{c}{ RVoG } & \multicolumn{2}{c}{ RVoG-vtd } & \multicolumn{2}{c}{ GRVoG-vtd } \\
\cline { 2 - 7 } & RMSE & Bias & RMSE & Bias & RMSE & Bias \\
\hline Sparse Savanna & 10.66 & 10.28 & $\mathbf{3 . 5 6}$ & $\mathbf{2 . 6 0}$ & 5.20 & 3.94 \\
Low Forest & $\mathbf{5 . 4 0}$ & 2.20 & 8.32 & -5.62 & 5.78 & $-\mathbf{1 . 0 3}$ \\
High Forest & 7.91 & -6.21 & 10.64 & -8.62 & $\mathbf{7 . 4 1}$ & $\mathbf{- 5 . 8 2}$ \\
\hline
\end{tabular}

\subsection{Discussions of Inversion Results}

As Figure 13 demonstrated, in the areas with vegetation less than $25 \mathrm{~m}$, the RVoGvtd and GRVoG-vtd models are more consistent with LiDAR-based vegetation height, while RVoG model has obvious overestimation. However, in the regions with over $25 \mathrm{~m}$ vegetation, RVoG and GRVoG-vtd models perform better, and RVoG-vtd underestimates significantly. The RVoG model has a best effect when the vegetation height is about $25 \mathrm{~m}$, but its weakness is the resolution of height. Generally, the inversion results of RVoG model are concentrated around $25 \mathrm{~m}$ for high forest. The GRVoG-vtd model has higher inversion 
accuracy and higher resolution, which can reflect the height fluctuation obviously. The reason is the coherence points are very close in the CUC plane when the amplitude is small, but in the amplitude-phase plane, the distances between the coherence points are more affected by coherence phase which can still be distinguishable, promising a higher resolution. However, the inversion will be more easily affected by noise correspondingly, so the inversion variance will be slightly larger. Although the RMSE of GRVoG-vtd is the smallest on the whole, it will also be slightly worse locally. In practice, when the coherence amplitude is small, the influence of coherence phase can be regarded as floating in a relatively small range, which results in the small difference between RMSEs of GRVoG-vtd and RVoG in dense forest regions as Table 2 presented.

From Figure 14 we can arrival at a similar conclusion. The estimations of RVoG-vtd and GRVoG-vtd are relatively accurate when the vegetation height is less than $25 \mathrm{~m}$. In addition, for the regions with vegetation height greater than $25 \mathrm{~m}$, the height estimation errors of all the three methods increase in different degrees. The RVoG model estimations gradually converge to a certain height, while RVoG-vtd and GRVoG-vtd models show relatively divergent estimations. The average vertical wavenumber $k_{z}$ is about 0.12 for the PolInSAR data. When the vegetation height is greater than $\pi / k_{z}$, i.e., $27 \mathrm{~m}$ in this case, the determination of ground phase would depend entirely on the distribution of coherences in different polarization states on the fitted line. The errors of ground phase extraction in the first two stages will be relatively large. Consequence, the inversion errors of these algorithms based on single-baseline PolInSAR data will be larger in the corresponding region. At the same time, $k_{z}$ also affects the resolution of vegetation height inversion. It can be seen from Figure 5 that with the increase of vegetation height, the corresponding coherence distribution becomes more concentrated. Therefore, when the vegetation height is too large, the small deviation of the complex coherences will cause a large error in the estimation of the vegetation height, which can be called the ill problem. In this regard, multi-baseline fusion is recommended to further solve the problem that different values of $k_{z}$ have different height precision for varying height intervals.

\section{Conclusions}

The RVoG model does not compensate for temporal decorrelation, hence the observed coherence amplitude will be substantially less than the theoretical calculation result. As a result, the vegetation height will be overestimated in most areas. When the three-stage inversion method is directly applied to the RVoG-vtd model, the phenomenon that one coherence point corresponds to multiple heights will appear which is also called ambiguous problem. The previous four-stage inversion algorithm was proposed to solve this problem while maintaining a low computational complexity. The height will be underestimated in some areas because only the coherence phase is used for estimation after the extinction coefficient is fixed. In the conventional three-stage process, the Euclidean distance in the CUC plane is used as the similarity measurement. However, in the CUC plane, the theoretical distribution curve of coherence is a high curvature manifold, so it is misleading to use Euclidean distance for the measurements of the similarity. Although the Euclidean distance between the two points is very close, they may be far away from each other on the manifold, and the corresponding vegetation height is also very different. Therefore, the Euclidean distance is not enough to truly reflect the similarity between the observed coherence and the theoretical coherence.

In view of the above problems, this paper extended the GRVoG-vtd model and the corresponding novel four-stage inversion algorithm. The GRVoG-vtd model focused on the random motion of scatterers to compensate for temporal decorrelation and took the random scatterer density distribution into account. The novel four-stage method extended the conventional three-stage method by an additional stage for vegetation species classification and real-value factor estimation. The vegetation height estimation stage was also modified by converting the Euclidean distance to the generalized distance, which provided a new idea to estimate heights in the amplitude-phase plane instead of the CUC plane. The height 
inversion experiments were conducted using PolInSAR image of Lope National Park, where the sparse savannas and dense forests are the dominant vegetations. The inversion results were illustrated by qualitative analysis and quantitative comparison. Through a series of experiments, this paper proved the rationality of the models and the feasibility of the inversion process. The comparison between the new model and the traditional models also showed the superiority of the GRVoG-vtd model.

Author Contributions: Conceptualization , C.X. and J.Y. (Jian Yang); methodology, C.X.; software, H.W.; validation, C.X., J.Y. (Jian Yang) and T.Z.; formal analysis, C.X.; investigation, C.X.; resources, J.Y. (Junjun Yin); data curation, C.X.; writing-original draft preparation, C.X.; writing-review and editing, T.Z.; visualization, C.X.; supervision, C.X. and L.Z.; project administration, J.Y. (Jian Yang); funding acquisition, J.Y. (Jian Yang). All authors have read and agreed to the published version of the manuscript.

Funding: This research was funded in part by National Key Research and Development Program of China grant number 2017YFB0502703 and by National Natural Science Foundation of China grant number 61771043.

Data Availability Statement: Restrictions apply to the availability of these data. Data was obtained from the European Space Agency and are available at https:/ / earth.esa.int/eogateway/campaigns / afrisar-2016 with the permission of the European Space Agency.

Acknowledgments: Thanks to DLR for providing PolInSAR data and LiDAR data, so that the new method can be tested, and the quantitative accuracy can be analyzed. Thanks to National Key Research and Development Program of China for funding the research. The authors would like to thank the anonymous reviewers for their constructive suggestions.

Conflicts of Interest: The authors declare no conflict of interest.

Abbreviations
$\begin{array}{ll}\text { The following abbreviations are used in this manuscript: } \\ \text { PolInSAR } & \text { Polarimetric interferometric synthetic aperture radar } \\ \text { LiDAR } & \text { Light detection and ranging } \\ \text { RVoG } & \text { Random volume over ground model } \\ \text { RVoG-vtd } & \text { Random volume over ground model with volumetric temporal decorrelation } \\ \text { GRVoG-vtd } & \text { Generalized RVoG-vtd } \\ \text { EM } & \text { Expectation-Maximum } \\ \text { DLR } & \text { German Aerospace Center } \\ \text { CUC } & \text { Complex unit circle } \\ \text { LUT } & \text { Look up table } \\ \text { GMM } & \text { Gaussian mixture model } \\ \text { RMSE } & \text { Root of mean square error }\end{array}$

\section{Appendix A. The Derivation of the GRVoG Model Function}

This section listed the main steps of the derivation of the GRVoG model function which have significant differences from the RVoG model. The article [7] can provide more details for readers really interested in the derivation. As in [7], the average field incident on the $\mathrm{j}$-th scatterer $\left\langle E\left(\mathbf{R}_{j}, \omega ; 1\right)\right\rangle$ satisfies:

$$
\left\langle E\left(\mathbf{R}_{j}, \omega ; 1\right)\right\rangle=A \exp \left(i k\left|\mathbf{R}_{j}-\mathbf{R}_{1}\right|\right)+\int \rho(\mathbf{R})\langle E(\mathbf{R}, \omega ; 1)\rangle\left\langle f\left(\widehat{R}_{1, R} ; \widehat{R}_{R, j}\right)\right\rangle \frac{\exp \left(i k\left|\mathbf{R}_{j}-\mathbf{R}\right|\right)}{\left|\mathbf{R}_{j}-\mathbf{R}\right|} d^{3} R
$$

where $\mathbf{R}_{j}$ and $\mathbf{R}_{1}$ represent the locations of the $j$-th scatterer and the transmitting radar, respectively. $f\left(\widehat{R}_{1, R} ; \widehat{R}_{R, j}\right)$ means the normalized, medium-ensemble-averaged scattering amplitude [34]. $\rho(\mathbf{R})$ is the scatterer density distribution function. $\omega$ and $k$ are the frequency and the wavenumber. 
Assuming the distribution of volume scatterer density is $\rho(h)$, the solution of the equation can be obtained by the stationary phase method [35]:

$$
\left\langle E\left(\mathbf{R}_{j}, \omega ; 1\right)\right\rangle=\operatorname{Aexp}\left[\left(i k\left|\mathbf{R}_{j}-\mathbf{R}_{1}\right|\right)+\frac{2 \pi i\left\langle f\left(\hat{R}_{1, R} ; \hat{R}_{R, j}\right)\right\rangle \int_{h_{v}-d_{j}}^{h_{v}} \rho(h) d h}{k \cos \theta_{j}}\right]
$$

where $h_{v}$ is the height of the forest in the area, $d_{j}$ is the depth of the j-th scatterer relative to the height of the tree, and $\theta_{j}$ is the angle between the line connecting the transmitting radar $\mathbf{R}_{1}$ and the scatterer position $\mathbf{R}_{j}$ and the vertical direction, i.e., the depression angle.

According to the same method, we can get:

$$
\left\langle E\left(\mathbf{R}_{1}, \omega ; j\right)\right\rangle=A^{2} f_{j}\left(\hat{R}_{1, j} ; \hat{R}_{j, 1}\right) \exp \left[2\left(i k\left|\mathbf{R}_{1}-\mathbf{R}_{j}\right|\right)+\frac{4 \pi i\left\langle f\left(\hat{R}_{j, 1} ; \hat{R}_{j, 1}\right)\right\rangle \int_{h_{v}-d_{j}}^{h_{v}} \rho(h) d h}{k \cos \theta_{j}}\right]
$$

After obtaining the single-frequency echo from single scatterer, it is necessary to integrate the response of the single scatterer over the entire bandwidth to obtain the overall echo signal of the scatterer. Furthermore, we add up the echoes of all the scatterers to get the expression:

$$
\left\langle E\left(\mathbf{R}_{\mathbf{1}}\right) E^{*}\left(\mathbf{R}_{\mathbf{2}}\right)\right\rangle \approx A^{4} \mathrm{e}^{j \phi_{0}} \int_{0}^{h_{v}} \exp \left(i k_{z} z\right) \rho(z) \exp \left[\frac{-2 \kappa_{e} \int_{z}^{h_{v}} \rho(h) d h}{\cos \theta}\right] d z
$$

with $\kappa_{e}$ is the vegetation extinction coefficient defined as:

$$
\kappa_{e}=\frac{4 \pi \rho_{0} \operatorname{Im}\langle f\rangle}{k}
$$

Finally, the complex coherence is normalized, i.e.:

$$
\frac{\left\langle E\left(\mathbf{R}_{\mathbf{1}}\right) E^{*}\left(\mathbf{R}_{\mathbf{2}}\right)\right\rangle}{\sqrt{\left|E\left(\mathbf{R}_{\mathbf{1}}\right)\right|^{2}\left|E\left(\mathbf{R}_{\mathbf{2}}\right)\right|^{2}}}=\mathrm{e}^{j \phi_{0}} \frac{\int_{0}^{h_{v}} \exp \left(j k_{z} z\right) \rho(z) \exp \left(\frac{\left.-2 \kappa_{e} \int_{z}^{h_{v}} \rho(h) d h\right)}{\cos \theta}\right) d z}{\int_{0}^{h_{v}} \rho(z) \exp \left(\frac{\left.-2 \kappa_{e} \int_{z}^{h_{v}} \rho(h) d h\right)}{\cos \theta}\right) d z}
$$

\section{References}

1. Cloude, S.R.; Papathanassiou, K.P. Polarimetric optimisation in radar interferometry. Electron. Lett. 1997, 33, 1176-1178. [CrossRef]

2. Papathanassiou, K.P.; Cloude, S.R. Single-baseline polarimetric SAR interferometry. IEEE Trans. Geosci. Remote Sens. 2001, 39, 2352-2363. [CrossRef]

3. Cloude, S.R.; Papathanassiou, K.P. Polarimetric SAR Interferometry. IEEE Trans. Geosci. Remote Sens. 1998, 36, 1551-1565. [CrossRef]

4. Yamada, H.; Sato, K.; Yamaguchi, Y.; Boerner, W.M. Interferometric phase and coherence of forest estimated by ESPRIT-based polarimetric SAR interferometry. In Proceedings of the IEEE International Geoscience and Remote Sensing Symposium, Toronto, ON, Canada, 24-28 June 2002.

5. Ballester-Berman, J.D.; Lopez-Sanchez, J.M. Applying the Freeman-Durden Decomposition Concept to Polarimetric SAR Interferometry. IEEE Trans. Geosci. Remote Sens. 2009, 48, 466-479. [CrossRef]

6. Aghababaee, H.; Sahebi, M.R. Model-Based Target Scattering Decomposition of Polarimetric SAR Tomography. IEEE Trans. Geosci. Remote Sens. 2018, 56, 972-983. [CrossRef]

7. Treuhaft, R.N.; Madsen, S.N.; Moghaddam, M.; Van Zyl, J.J. Vegetation characteristics and underlying topography from interferometric radar. Radio Sci. 1996, 31, 1449-1485. [CrossRef]

8. Treuhaft, R.N.; Siqueira, P.R. Vertical structure of vegetated land surfaces from interferometric and polarimetric radar. Radio Sci. 2016, 35, 141-177. [CrossRef]

9. Lu, H.; Suo, Z.; Guo, R.; Bao, Z. S-RVoG model for forest parameters inversion over underlying topography. Electron. Lett. 2013, 49, 618-619. [CrossRef]

10. Sun, X.; Wang, B.; Xiang, M.; Fu, X.; Li, Y. S-RVoG Model Inversion Based on Time-Frequency Optimization for P-Band Polarimetric SAR Interferometry. Remote Sens. 2019, 11, 1033. [CrossRef] 
11. Sun, X.; Wang, B.; Xiang, M.; Jiang, S.; Fu, X. Forest Height Estimation Based on Constrained Gaussian Vertical Backscatter Model Using Multi-Baseline P-Band Pol-InSAR Data. Remote Sens. 2018, 11, 42. [CrossRef]

12. Wang, X.; Xu, F. A PolinSAR Inversion Error Model on Polarimetric System Parameters for Forest Height Mapping. IEEE Trans. Geosci. Remote Sens. 2019, 57, 5669-5685 [CrossRef]

13. Bryan, R.; Michael, D.; Marco, L. Uncertainties in Forest Canopy Height Estimation From Polarimetric Interferometric SAR Data IEEE J. Select. Top. Appli. Earth Observat. Remote Sens. 2018, 11, 1-14.

14. Cloude, S.R.; Papathanassiou, K.P. Three-stage inversion process for polarimetric SAR interferometry. IEE Proc. Radar Sonar Navigat. 2003, 150, 125-134. [CrossRef]

15. Managhebi, T.; Maghsoudi, Y.; Zoej, M.J.V. A Volume Optimization Method to Improve the Three-Stage Inversion Algorithm for Forest Height Estimation Using PolInSAR Data. IIEEE Trans. Geosci. Remote Sens. Lett. 2018, 15, 1-5. [CrossRef]

16. Ballester-Berman, J.D.; Vicente-Guijalba, F.; Lopez-Sanchez, J.M. A Simple RVoG Test for PolInSAR Data. IEEE J. Select. Top. Appl. Earth Observat. Remote Sens. 2017, 8, 1028-1040. [CrossRef]

17. Ghasemi, N.; Tolpekin, V.A.; Stein, A. Estimating Tree Heights Using Multibaseline PolInSAR Data With Compensation for Temporal Decorrelation, Case Study: AfriSAR Campaign Data. IEEE J. Select. Top. Appl. Earth Observat. Remote Sens. 2018, 11, 3464-3477. [CrossRef]

18. Brigot, G.; Simard, M.; Colin-Koeniguer, E.; Boulch, A. Retrieval of Forest Vertical Structure from PolInSAR Data by Machine Learning Using LIDAR-Derived Features. Remote Sens. 2019, 11, 381. [CrossRef]

19. Sun, X.; Wang, B.; Xiang, M.; Zhou, L.; Jiang, S. Forest Height Estimation Based on P-Band Pol-InSAR Modeling and Multi-Baseline Inversion. Remote Sens. 2020, 12, 1319. [CrossRef]

20. Kumar, P.; Krishna, A.P. InSAR-Based Tree Height Estimation of Hilly Forest Using Multitemporal Radarsat-1 and Sentinel-1 SAR Data. IEEE J. Select. Top. Appl. Earth Observat. Remote Sens. 2020, 12, 5147-5152. [CrossRef]

21. Xie, Y.; Fu, H.; Zhu, J.; Wang, C.; Xie, Q. A LiDAR-Aided Multibaseline PolInSAR Method for Forest Height Estimation: With Emphasis on Dual-Baseline Selection. IEEE Geosci. Remote Sens. Lett. 2019, 17, 1-10. [CrossRef]

22. Pourshamsi, M.; Garcia, M.; Lavalle, M.; Balzter, H. A Machine-Learning Approach to PolInSAR and LiDAR Data Fusion for Improved Tropical Forest Canopy Height Estimation Using NASA AfriSAR Campaign Data. IEEE J. Select. Top. Appl. Earth Observat. Remote Sens. 2018, 11, 3453-3463. [CrossRef]

23. Hajnsek, I.; Kugler, F.; Lee, S.K.; Papathanassiou, K.P. Tropical-Forest-Parameter Estimation by Means of Pol-InSAR: The INDREXII Campaign. IEEE Trans. Geosci. Remote Sens. 2009, 47, 481-493. [CrossRef]

24. Zebker, H.A.; Villasenor, J. Decorrelation in interferometric radar echoes. IEEE Trans. Geosci. Remote Sens. 1992, 30, 950-959. [CrossRef]

25. Lavalle, M.; Khun, K. Three-Baseline Approach to Forest Tree Height Estimation. In Proceedings of the Eusar European Conference on Synthetic Aperture Radar, Berlin, Germany, 3-5 June 2014.

26. Lavalle, M. A Temporal Decorrelation Model for Polarimetric Radar Interferometers. IEEE Trans. Geosci. Remote Sens. 2012, 50, 2880-2888. [CrossRef]

27. Simard, M.; Denbina, M. An Assessment of Temporal Decorrelation Compensation Methods for Forest Canopy Height Estimation Using Airborne L-Band Same-Day Repeat-Pass Polarimetric SAR Interferometry. IEEE J. Select. Top. Appl. Earth Observat. Remote Sens. 2018, 11, 95-111. [CrossRef]

28. Tayebe, M.; Yasser, M.; Mohammad, V.Z. Four-Stage Inversion Algorithm for Forest Height Estimation Using Repeat Pass Polarimetric SAR Interferometry Data. Remote Sens. 2018, 10, 1174.

29. Pardini, M.; Tello, M.; Cazcarra-Bes, V.; Papathanassiou, K.P.; Hajnsek, I. L- and P-Band 3-D SAR Reflectivity Profiles Versus Lidar Waveforms: The AfriSAR Case. IEEE J. Select. Top. Appl. Earth Observat. Remote Sens. 2018, 11, 3386-3401. [CrossRef]

30. Ngo, Y.N.; Minh, D.H.T.; Moussawi, I.; Villard, L.; Toan, T.L. Afrisar-Tropisar: Forest Biomass Retrieval by P-Band Sar Tomography. In Proceedings of the IGARSS 2018 IEEE International Geoscience and Remote Sensing Symposium, Valencia, Spain, 22-27 July 2018.

31. Moussawi, I.E.; Minh, D.H.T.; Baghdadi, N.; Abdallah, C.; Strauss, O. L-Band Uavsar Tomographic Imaging in Dense Forest: Afrisar Results. In Proceedings of the IGARSS 2018 IEEE International Geoscience and Remote Sensing Symposium, Valencia, Spain, 22-27 July 2018.

32. Technical Assistance for the Development of Airborne SAR and Geophysical Measurements during the AfriSAR Experiment. Available online: https://earth.esa.int/eogateway/documents/20142/37627/AfriSAR-Final-Report.pdf (accessed on 24 December 2020).

33. Kugler, F.; Lee, S.K.; Hajnsek, I.; Papathanassiou, K.P. Forest Height Estimation by Means of Pol-InSAR Data Inversion: The Role of the Vertical Wavenumber. IEEE Trans. Geosci. Remote Sens. 2015, 53, 5294-5311. [CrossRef]

34. Lang, R.H. Electromagnetic backscattering from a sparse distribution of lossy dielectric scatterers. Radio Sci. 1981, 16, 15-30. [CrossRef]

35. Ishimaru, A. Wave Propagation and Scattering in Random Media; Academic Press: New York, NY, USA, 1978. 